\title{
Towards democratizing and automating online conferences: lessons from the Neuromatch Conferences
}

Titipat Achakulvisut, ${ }^{1}$ Tulakan Ruangrong, ${ }^{2}$ Patrick Mineault, Tim P. Vogels, ${ }^{3}$ Megan A. K. Peters, ${ }^{4}$ Panayiota Poirazi ${ }^{5}$ Christopher Rozell, ${ }^{6}$ Brad Wyble, ${ }^{7}$ Dan F. M. Goodman, ${ }^{8}$ and Konrad Paul Kording ${ }^{1}$

${ }^{1}$ Department of Bioengineering, University of Pennsylvania, Philadelphia, United States

${ }^{2}$ Department of Biomedical Engineering, Mahidol University, Nakhon Pathom, Thailand

${ }^{3}$ The Institute of Science and Technology Austria, Klosterneuburg, Austria

${ }^{4}$ Department of Cognitive Sciences, University of California, Irvine, Irvine, CA, United States

${ }^{5}$ Institute of Molecular Biology and Biotechnology (IMBB), Foundation for Research and Technology-Hellas (FORTH), Crete, Greece.

${ }^{6}$ School of Electrical and Computer Engineering, Georgia Institute of Technology

${ }^{7}$ Department of Bioengineering and the Department of Psychology, Penn State University, United States

${ }^{8}$ Department of Electrical and Electronic Engineering, Imperial College London, United Kingdom

Correspondence: titipata@seas.upenn.edu (T. Achakulvisut), d.goodman@imperial.ac.uk (D. Goodman), bwyble@gmail.com (B.Wyble), koerding@gmail.com (K. Kording)

\section{Abstract}

Legacy conferences are costly, time-consuming, and exclude scientists lacking various resources or abilities. During the 2020 pandemic, we created an online conference platform, Neuromatch Conferences, aimed at developing technological and cultural changes to make conferences more democratic, scalable, and accessible. We discuss the lessons we learned.

\section{Neuromatch conferences}

Conferences are a backbone of scholarly communication, where scientists can learn and share about new ideas, meet colleagues and make friendships. However, the need for travel and physical infrastructure means conferences are expensive and time-consuming, they contribute to global climate change [1], and exclude scientists for many reasons (e.g., budgetary [2], mobility, $[3,4]$ or size constraints).

We created the Neuromatch Conferences (NMC, https://neuromatch.io) [5] to make conferences more climate-friendly [1], accessible [6], and democratic [7]. We held three events in March, May, and October 2020. Similar to legacy conferences, scientists gave and viewed talks, as well 
as networked in a variety of ways. NMC grew with each iteration. NMC3 featured more than 900 talks and approximately 4700 attendees while being driven by a small team of volunteers, so we had to develop technology solutions to enable this growth. Replacing the traditional review process where only few submissions are selected for talks and most are delegated to posters or rejected, we opted for a light-handed editorial approach focused on scheduling rather than evaluation: All submissions were accepted for talks, and each talk was arranged at a time that maximized live audiences in all time zones. To help attendees select their program, we relied on a combination of search and recommendation algorithms. We also used recommendation engines to kickstart online socialisation by introducing scientists to one another. To promote openness and inclusivity [8], NMC3 featured several curated events focused on both inclusion issues in various communities such as Black In Neuro (https://www.blackinneuro.com), Queer In Neuro (https://twitter.com/queerinneuro), and first generation students, as well as professional development issues such as science communication and future funding priorities.

\section{Towards improving on legacy conferences}

\section{Use data to make online conferences more democratic and scalable}

We created an automatic system for dealing with talk scheduling and agenda curation (Figure 1A). We asked attendees and presenters for their preferred times. After abstract submission closed, we evaluated each talk's expected audience by asking participants to choose talks. This happened double-blind because we wanted attendees to choose interesting science without regard to authors' status. We supplemented this interest-based assessment with a recommendation algorithm [9] that suggested additional talks based on other participants' choices and similarity of abstracts (Figure 1B). We debuted this solution for NMC3 and received more than 23,000 votes from 1,231 attendees. With these votes, we could quickly build a picture of likely audiences (i.e., who wanted to see what and when) and which talks should not overlap.

Next, we employed a custom-made, two stage scheduling algorithm (Figure 1C). In the first stage, we grouped talks into sessions by their indicated availability. From the possible sets of sessions, we selected those which maximized session coherence based on audience overlap and topic similarity [9]. In the second scheduling stage we assigned time slots to each session. We used an integer linear programming (ILP) approach [10] to maximize the total number of expected watchhours for each session, assuming higher likelihood of watching a session if it contained multiple interesting talks and if assigned timeslots overlapped with core watch-hours in the timezones of the target audience (i.e., not in the middle of the night). We believe that automatic scheduling is useful but there is a challenging tradeoff between enabling scientists to participate and the desire to have maximal audiences.

Crucially, we could automate many other aspects of the organizational workflow. For example, speaker communications often happened via automated emails with relevant information and calendar links, and participants received talk suggestions that were relevant to their interests. Automation is needed to allow a small team to run large conferences. 
A

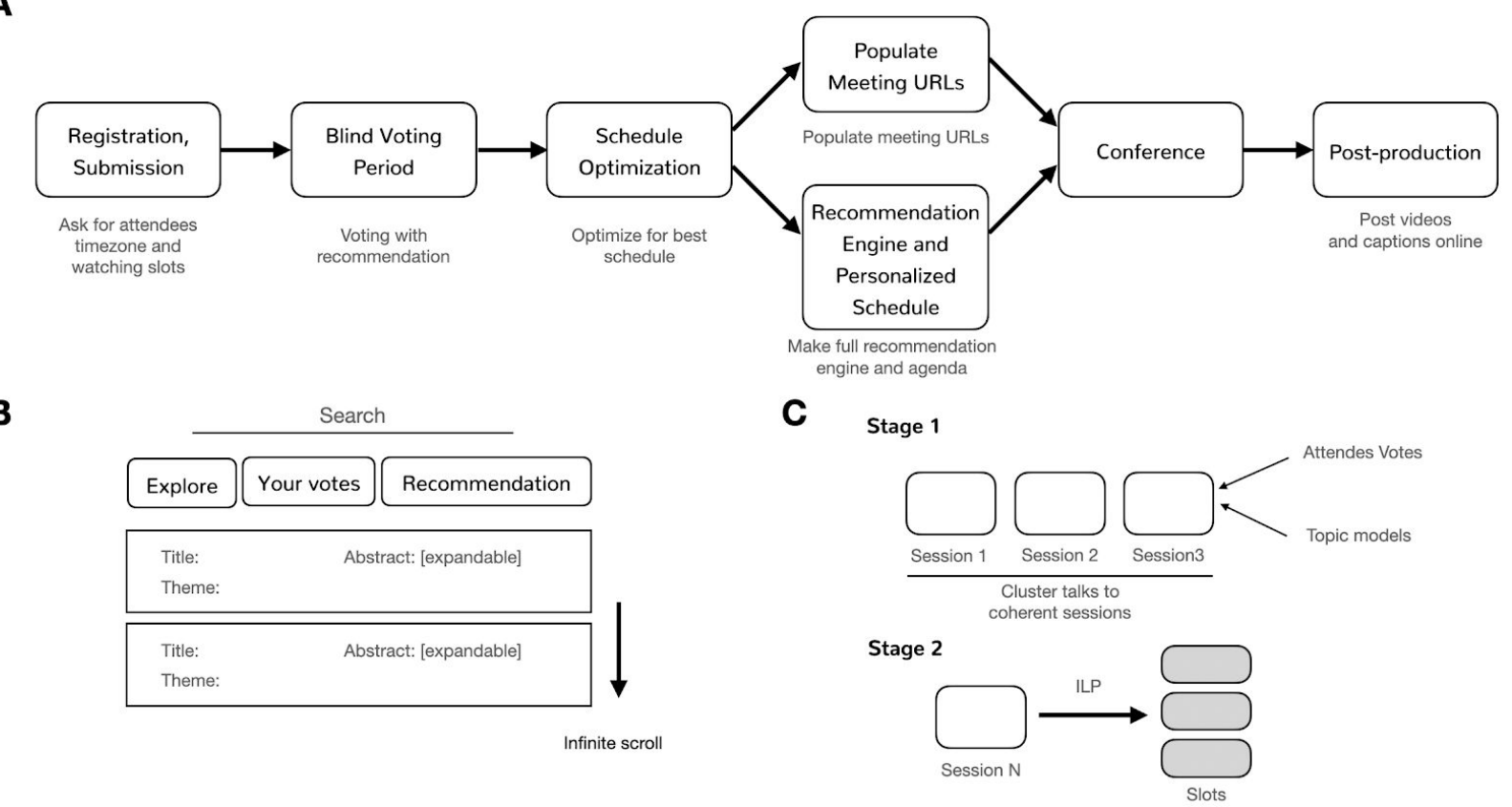

Figure 1. Schematic of the agenda building and talk scheduling. A. Conference timeline We opened for registration and submission followed by the blind voting using our recommendation engine. We then ran the schedule optimization so as to maximize the number of attendees for each talk. After the conference, we populated the agenda and URLs followed by post-production and releasing videos after the conference. B. Schematic of a recommendation engine for voting. We had a default tab for exploring, storing votes, and recommending similar abstracts. The recommendation tab returns abstracts related to the votes ranked by topical distance. Users can vote for abstracts that they would like to see and their selections are stored on Your votes tab. C. Schematic of how we solved talk scheduling. We first grouped submissions into sessions using votes and topic similarity. We then applied ILP to assign time slots to each session.

\section{Improve inclusivity and sustainability}

Towards this goal, our editorial process filtered out only non-science and off-topic submissions. We worked hard to achieve gender equality [11] at NMC, with approximately $50 \%$ female speakers at each NMC.

The ratio of female-to-male participants at all career stages roughly matched those of the field as a whole, as estimated by BiasWatchNeuro (https://biaswatchneuro.com/base-rates/neuroscience-base-rates). The "leaky pipeline" [12] is clearly apparent, with a majority of undergraduates female, and worsening ratios at each consecutive career stage (Figure 2). We believe that, in part due to the democratization of science and the low financial and logistical barriers to participation, online conference formats can attract a more diverse set of attendees than legacy conferences, and may help to 'plug' the leaky pipeline. However, further data and research are needed to validate that this is actually the case. 

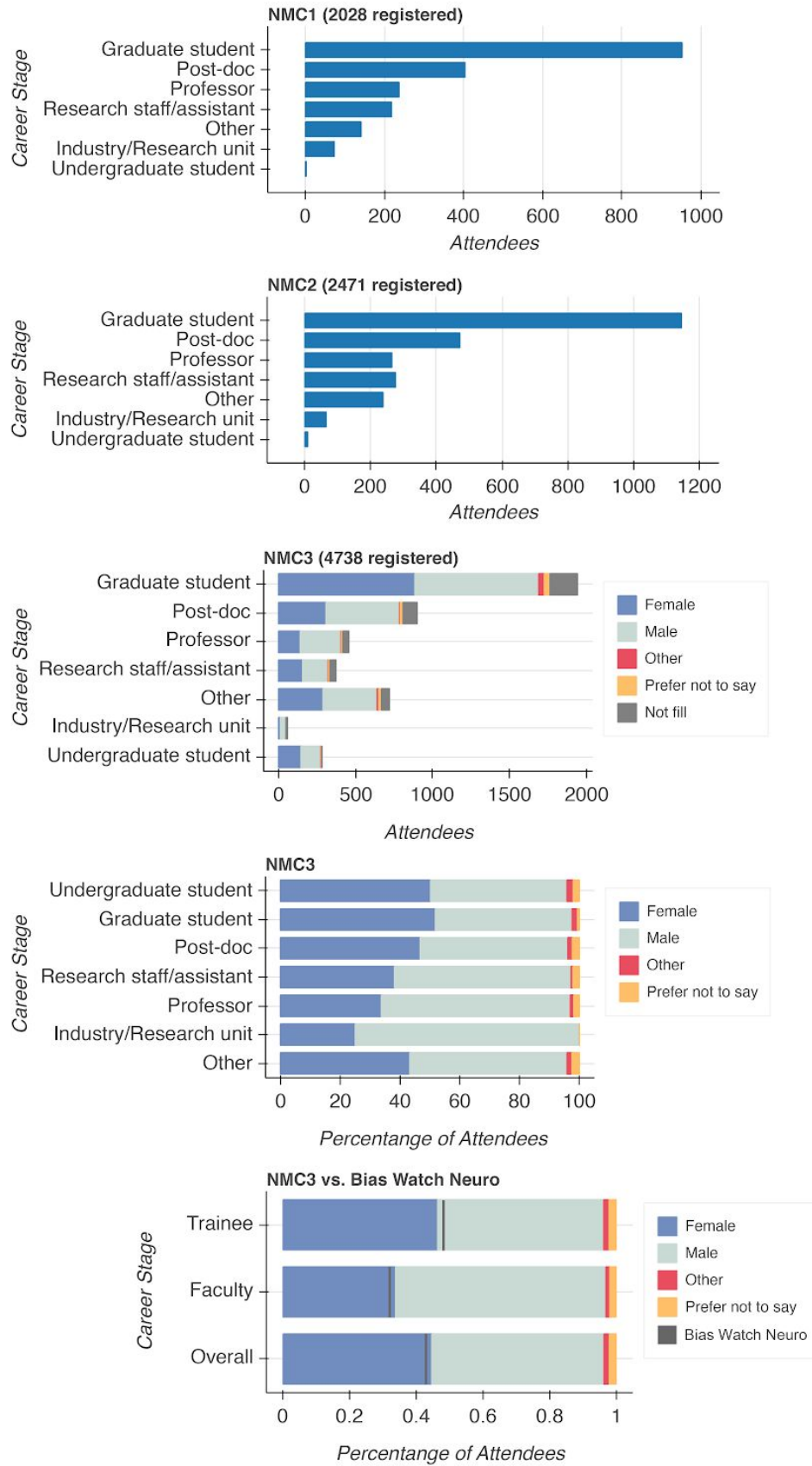

Figure 2. Number of registered attendees for each NMC edition. Registrations for each NMC edition career stage and by gender in NMC3. Overall, NMCs brought diverse participants in both career stages and gender. In NMC3, we observed a higher percentage of female-to-male participants in early career stages but this was reversed in the postdoc, professor, industry, and research units. Our participants female-to-male trainee (graduate student and postdoc), faculty, and overall ratios are $48 \%$, $35 \%$, and $46 \%$, roughly matching the estimates for neuroscience as a whole of $50 \%, 33 \%$, and $44 \%$ estimated by Bias Watch Neuro. 


\section{Lessons from organizing online conferences}

\section{Online conferences can be cheap}

Online streaming is relatively cheap. We spent a total of around $\$ 1000$ for Crowdcast for the first two conferences and $\$ 4000$ for NMC3 for Zoom Webinar. Storing data and serving our website comes at nominal costs [5]. While NMC 1 and 2 were free, we started charging a waivable $\$ 25$ waivable fee for participation in NMC3, hoping to put the conference on a sustainable path [2]. Indeed, we were able to collect all streaming costs of NMC3 from attendance fees. Algorithms enable major time and money savings, e.g. when it comes to making schedules and their development, algorithms promise to do it better and faster than volunteers. To facilitate scale-up, more automation is needed so as to minimize administrative, editorial and program committees' work.

\section{Streaming platforms affect user experience}

NMC1 and NMC2 focused mainly on computational neuroscience. For both, we had a main track with keynotes and submitted talks with 5 parallel tracks, delivered via zoom at NMC1 and Crowdcast (CC) at NMC2. Talks were available for later viewing, and audience feedback indicated that interactions between speaker and participants were good. Unfortunately, CC audio-visual and streaming quality was inconsistent, and speakers had to re-start their browser frequently. For NMC3, we expanded the conference to serve a broader range of neuroscience with 6 themes and up to 9 parallel tracks. We opted for Zoom webinar as our streaming platform, which offered more reliable service, but lagged in user engagement. As the choice of streaming software profoundly affects the user experience, we still look for solutions that offer both an interactive user interface and stable high-resolution streaming.

\section{Hosting and distributing videos after the conference}

Hosting NMC3 required approximately 160 volunteers to host the set of approximately 900 events. We sent calendar links to hosts to make sure they got correct time with detailed instructions. A live chat backbone (on Slack) was essential for addressing last-minute cancellations, no-shows, and technical problems. During NMC1 and NMC2, the hosts spent much of their time making sure that speakers knew where and when to show up and we experienced considerable drop-out. Thanks to hosting each session with two hosts, one for the technical and one for the introductions, NMC3 ran efficiently and reliably, despite having 10 times more talks than the previous NMC. After NMC3, each talk was posted separately on YouTube, with labels and links to the original program. With this model the conference event is no longer the ephemeral end product. Rather, it includes the delivery of the scientific content in a durable and accessible long-term format. We believe that all online conferences have a duty to do so.

Online conferences should facilitate social interactions: The need to replace poster session 
In legacy conferences, much of the social interaction occurs during the coffee break and poster presentations. In online conferences, an important aspect is to ensure that the agenda and platform provide enough social interaction. NMC uses machine learning algorithms to help match attendees for one-on-one meetings similar to the coffee break [13]. We tried to create the equivalent poster interaction online using two formats. In NMC2, we created a poster session allowing attendees to visit common sessions, view the content, and ask questions. Unfortunately, attendance was generally low. Feedback suggests that bad timing and low visibility were part of the issue. However, the problem of low poster attendance seems to be common with other online conferences [14]. To improve attendee experience at NMC3, we created "interactive talks" instead of posters: 5 minute talks followed by 10 minutes of discussion. From our experience, these interactive talks fostered more interaction compared to our previous poster sessions, but still failed to capture the real poster experience. It remains to be seen whether a poster-like format with free-wheeling personal interaction can successfully be transferred to the online space. Technical solutions regarding proximity-graded sharing of virtual spaces such as GatherTown or Mozilla Hubs have potential here, but more innovation is needed. Making online conferences feel great and personal is the ultimate goal that we need to innovate towards.

\section{Conclusion}

Once the technical issues are solved, online conferences can be organized by relatively small teams. Our experience, and that of others such as Federation of European Neuroscience Society Forum 2020 (https://forum2020.fens.org/), Organization for Human Brain Mapping (https://www.humanbrainmapping.org/), ICLR 2020 [15], NeurIPS 2020 [16], has shown that online conferences have great potential to establish a forum for scientific exchange with numerous advantages to physical meetings: less costly, environmentally friendly, more inclusive and largely interactive. The Neuromatch Conferences serve as proof that moving conferences online can alleviate inclusivity issues associated by reducing the barriers to participation for people from many areas of the world [6], people who cannot afford expensive accommodation and registration, those subject to travel restrictions, or those with caring responsibilities who cannot leave for long periods of time.

\section{Acknowledgement}

We thank all of our volunteers from the NMC conferences (list of names in the appendix). We also thank the NSF for support from 1734220 to B.W., and DARPA for support to T.A.

\section{References}

1 Burtscher, L. et al. (2020) The carbon footprint of large astronomy meetings. Nat. Astron. 4, 823-825 
2 Bottanelli, F. et al. (2020) Science during lockdown - from virtual seminars to sustainable online communities. J. Cell Sci. 133, jcs249607

3 De Picker, M. (2020) Rethinking inclusion and disability activism at academic conferences: strategies proposed by a PhD student with a physical disability. Disabil. Soc. 35, 163-167 4 Weissgerber, T. et al. (2020) Mitigating the impact of conference and travel cancellations on researchers' futures. eLife 2020;9:e57032

5 Achakulvisut, T. et al. (2020) Improving on legacy conferences by moving online. eLife 2020;9:e57892

6 Sarabipour, S. (2020) Virtual conferences raise standards for accessibility and interactions. eLife 2020;9:e62668

7 Rich, S. et al. (2020) Out of COVID, a conference: lessons from creating a new, free, and entirely virtual academic meeting amidst a pandemic. DOI: 10.31219/osf.io/6f7pa

8 Martinez-Acosta, V.G. and Favero, C.B. (2018) A Discussion of Diversity and Inclusivity at the Institutional Level: The Need for a Strategic Plan. J. Undergrad. Neurosci. Educ. 16, A252-A260

9 Achakulvisut, T. et al. (2016) Science Concierge: A fast content-based recommendation system for scientific publications. PLoS One 11, e0158423

10 Taylor, C.J. (2008) On the Optimal Assignment of Conference Papers to Reviewers. at <https://repository.upenn.edu/cgi/viewcontent.cgi?article=1934\&context=cis reports>

11 Nature (2016) Women need to be seen and heard at conferences. Nature 538, 290

12 Clark Blickenstaff*, J. (2005) Women and science careers: leaky pipeline or gender filter? Gend. Educ. 17, 369-386

13 Achakulvisut, T. et al. (2020) neuromatch: Algorithms to match scientists. eLife Labs at <https://elifesciences.org/labs/5ed408f4/neuromatch-algorithms-to-match-scientists>

14 Bozelos, P.A. and Vogels, T.P. (2020) Talking science, online. Nat. Rev. Neurosci. DOI: 10.1038/s41583-020-00408-6

15 ICLR Organizing Committees, Gone Virtual: Lessons from ICLR2020. [Online]. Available: https://medium.com/@iclr_conf/gone-virtual-lessons-from-iclr2020-1743ce6164a3. [Accessed: 26-Nov-2020]

16 Rush, A. M., \& Strobelt, H. (2020). MiniConf--A Virtual Conference Framework. arXiv preprint arXiv:2007.12238.

\section{Appendix}


Below is a list of Neuromatch Conferences volunteers.

Adam Steel, Adeel Razi, Aditya lyer, Adrienne Fairhall, Aina Puce, Aiying Zhang, Alberto Antonietti, Alex Hernandez-Garcia, Alexander Arguello, Alexander Gonzalez, Alexandra Tzilivaki, Ali Rigby, Ameet Annasaheb Rahane, Amita Kapoor, Andre Fenton, Angela Hwang, Anqi Wu, Arash Ash, Arianna Dorschel, Athanasia Papoutsi, B. Ezgi Arikan, Ben Evans, Benjamin Kanter, Byron V. Galbraith, Carsen Stringer, Cheng Xue, Chloe Callahan-Flintoft, Chris Rozell, Christoph Metzner, Daan Wesselink, Damion V. Demeter, Davide Valeriani, Denis Jaubaudon, Devapratim Sarma, Dimitra Maoutsa, Doby Rahnev, Ehsan Rezayat, Ekaterina Morozova, Elaine Corbett, Elenor Morgenroth, Elisabeta Balla , Ellie Morgenroth, Elnaz Alikarami, Emily Aery Jones, Felipe Parodi, Filip Morys, Forouzan Farahani, G. Lopez Bendito, Gaia Molinaro, Gaia Novarino, Gaiqing Kong, Gelana Tostaeva, Gowri Somasekhar, Gunnar Blohm, Gunnar Schmidtmann, Gyorgy Lur, Hadi K Motamedi, Haydee Garcia Lazaro, Huriye Atilgan, Ignacio Obeso, Ilenna Jones, Ishaant Agarwal, Jackie Schiller, James Finley, Jeremy Forest, John Butler, Jonathan Perdomo, Junda Zhu, Kalina Michalska, Kami Salibayeva, Karen Rommelfanger, Keerthi Krishnan, Kevin Wayne Rusch, Leena Ali ibrahim, Lei Zhang, Lena Ting, Leyla Loued-Khenissi, Lorenzo Fontolan, Madineh Sedigh-Sarvestani, Manu Madhav, Manuel Mameli, Manusmriti Agarwal, Marco Simões, Maria Reva, Marius 't Hart, Matteo Mancini, Melvin Selim Atay, Mengsen Zhang, Miguel Blacutt, Nidhi Seethapathi, Nikola Jajcay, Pavithra Rajeswaran, Rekha Varrier, Richard Lange, Richard Naud, Robert Froemke, Robert Rosenbaum, Roozbeh Farhoodi, Rosa Cossart, Ryan O'Donnell, Saeed Salehi, Sankaraleengam Alagapan, Sara Goering, Sean Escola, Sean K. Martin, Selim Melvin Atay, Shekoofeh Hedayati, Shlomit Beker, Shreya Jain, Siddharth Suresh, Silvia Pittolo, Sofie Van Den Bossche, Sophia Batchelor, Srikanth Ramaswamy, Stephanie Noble, Steven Peterson, Taha Morshedzadeh, Tara van Viegen, Tijl Grootswagers, Tom Burns, Trisha Kesar, Ulrik R Beierholm, Ute Habel, Vahid Esmaeili, Valentina Borghesani, William George Chapman, Xiqin LIU, Yalda Mohsenzadeh, Yang Chu, Yueqi Guo, Zeinab Esmaeilpour 